\title{
Infektionen des oberen Respirationstrakts bei Schildkröten
}

\author{
Ekaterina Kolesnik, Rachel E. Marschang
}

Erkrankungen der oberen Atemwege (URTD = upper respiratory tract disease) sind eines der häufigsten Probleme bei Schildkröten. Rhinitis, Konjunktivitis, Stomatitis und Glossitis werden regelmäßig beobachtet. Die Symptome reichen von Anorexie und allgemeiner Lethargie bis hin zu Lidödemen sowie Augen- und Nasenausfluss. Als Ursache werden vor allem Mykoplasmeninfektionen angesehen. Differenzialdiagnostisch sollten auch Herpes- oder Ranaviren in Betracht gezogen werden. Seltener und nur bei bestimmten Spezies sind auch Picornaviren und intranukleäre Kokzidien möglich. Zusätzlich spielen Hygiene, Temperatur und Luftfeuchtigkeit sowie fakultativ pathogene Bakterien und Pilze eine Rolle.

\section{Mykoplasmen}

Mykoplasmen werden vor allem bei Landschildkröten häufig beschrieben, in den letzten Jahren aber auch vermehrt bei Wasserschildkröten.

\section{Landschildkröten}

Die bisher nachgewiesenen Erreger lassen sich in 3 verschiedene Spezies einteilen:

- Mycoplasma agassizii

- Mycoplasma testudineum

- Mycoplasma testudinis

Mycoplasma (M.) agassizii ist assoziiert mit Erkrankungen des oberen Respirationstrakts ( Abb.1) und wird regelmäßig bei verschiedenen Landschildkröten-Spezies nachgewiesen. Am häufigsten finden sie sich bei Gopherschildkröten (Gopherus agassizii, G. polyphemus) in den USA und Testudo spp. in Europa.

M. testudineum wurde bei Gopherschildkröten in den USA nachgewiesen und ist ebenfalls mit Erkrankungen der oberen Atemwege assoziiert. Der Erreger scheint jedoch eine geringere Pathogenität aufzuweisen als M. agassizii.

M. testudinis war die erste Mykoplasmenspezies, die bei Schildkröten beschrieben wurde. Der Nachweis gelang aus der Kloake einer klinisch gesunden Maurischen Landschildkröte (Testudo graeca). Diese Mykoplasmenspezies ist nicht mit klinischen Symptomen assoziiert.

\section{Wasserschildkröten}

Mykoplasmen wurden auch bei Wasserschildkröten beschrieben, v.a. in verschiedenen Spezies der Neuwelt-Sumpfschildkröten (Familie Emydidae). Die Tiere zeigten entweder URTD-ähnliche Symptome oder waren klinisch gesund. Es ist zu erwarten, dass diese Bakterien auch bei anderen Spezies vorkommen und zum Teil zur Normalflora gehören, da häufig hohe Prävalenzen beschrieben werden (40-70\%).

\section{Symptomatik}

Durch Infektionsversuche mit M. agassizii an Gopherschildkröten konnten Erkrankungen der oberen Atemwege bei infizierten Tieren ausgelöst und somit

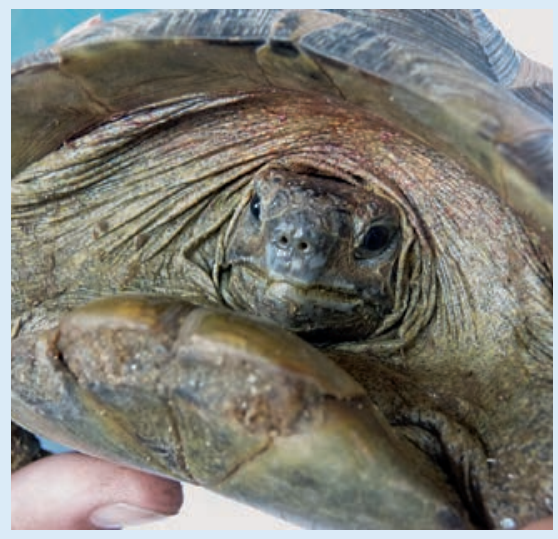

Abb. 1 Testudo graeca mit deutlicher Rhinitits aufgrund einer Mycoplasmeninfektion. die Henle-Koch'schen-Postulate erfüllt werden. Jedoch scheint die Ausprägung der klinischen Symptome sehr unterschiedlich zu sein. Infektionen mit Mykoplasmen reichen von klinisch unauffälligen Tieren bis hin zu schweren respiratorischen Erkrankungen. Bei chronischen Mykoplasmeninfektionen können klinische Symptome intermittierend auftreten und beispielsweise durch Stresssituationen verstärkt werden. Subklinisch infizierte Tiere stellen somit ein Erregerreservoir dar und können den Erreger unbemerkt in einem Bestand oder einer Population verbreiten.

\section{Herpesviren}

Herpesviren bei Schildkröten verursachen hauptsächlich eine diphtheroidnekrotisierende Stomatitis ( $\triangleright$ Abb.2), können sich aber auch in Glossitis ( Abb.3), Rhinitis, Dyspnoe und Konjunktivitis zeigen. Fallberichte mit Hepatitis und Meningoenzephalitis sind ebenfalls beschrieben.

\section{Landschildkröten}

Bei Landschildkröten werden 4 verschiedene Herpesvirustypen unterschieden: Testudinid Herpesvirus 1-4 (TeHV 1-4).

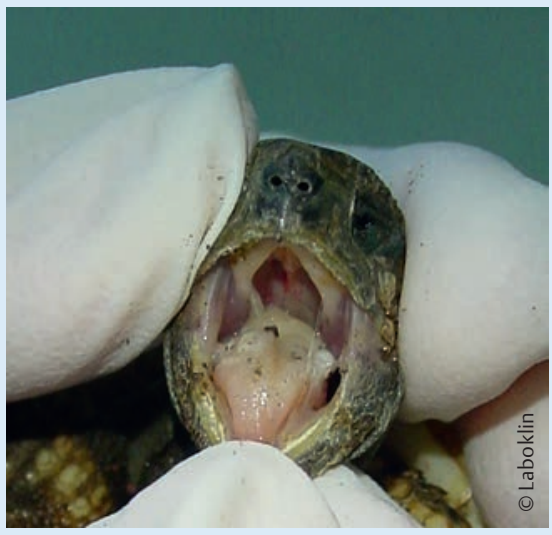

Abb. 2 Testudo hermanni mit einer diphtheroidnekrotisierenden Stomatitis durch eine Herpesvirusinfektion. 
In Europa hat TeHV 3 die größte Bedeutung und kommt vor allem bei europäischen Landschildkröten vor. Griechische (T. hermanni) und Russische Landschildkröten (T. horsfieldii) scheinen eine höhere Empfindlichkeit gegenüber TeHV $3 \mathrm{zu}$ besitzen als Maurische Landschildkröten.

TeHV 1 kommt vor allem bei Russischen Landschildkröten vor und gewinnt in Europa aufgrund des vermehrten Nachweises immer mehr an Bedeutung.

TeHV 2 kommt endemisch in Gopherschildkröten in Nordamerika vor. TeHV 4 wurde bisher nur in 2 Fallberichten beschrieben: bei einer klinisch unauffälligen Afrikanischen Schnabelbrustschildkröte (Chersina angulata) in den USA und einer Pantherschildkröte (Stigmochelys pardalis) mit respiratorischen Symptomen in Deutschland.

\section{Wasserschildkröten}

Bei Wasserschildkröten häufen sich vor allem in den letzten Jahren die Herpesviren-Nachweise. Die Benennung erfolgt in Anlehnung an die Spezies, in denen das Virus entdeckt wurde:

- Emydid Herpesviren 1-2

- Terrapene Herpesviren 1-2

- Glyptemys Herpesviren 1-2

- Pelomedusid Herpesvirus 1

\section{Ranaviren}

Infektionen mit Ranaviren zeigen sich bei Schildkröten ebenfalls mit Nasen- und Augenausfluss, ulzerativer Stomatitis mit käsigen Belägen, Konjunktivitis und subkutanen Ödemen im Halsbereich. Ranaviren wurden bei vielen verschiedenen Schildkrötenspezies nachgewiesen. Am häufigsten finden sie sich bei Dosen- und Schmuckschildkröten (Emydidae) und europäischen Landschildkröten (Testudo spp.), aber auch bei vielen anderen Arten.

Ranaviren sind bedeutende Krankheitserreger bei Amphibien. Sie kommen vor allem bei wildlebenden Amphibien vor. Somit ist eine Übertragung sowohl durch Wildtiere als auch durch Reptilien und Amphibien in Gefangenschaft möglich.

\section{Picornaviren}

Picornavirusinfektionen, auch als Virus „X“ oder Topiviren bekannt, sind mit verschiedenen klinischen Symptomen assoziiert. Am häufigsten gehen sie mit Panzererweichungen bei Jungtieren einher, vermutlich durch eine Nephropathie verursacht. Bei älteren Tieren sind Infektionen im Zusammenhang mit Stomatitis, Rhinitis, Pneumonie und Konjunktivitis, vereinzelt auch mit Enteritis und Aszites beschrieben.

Picornaviren konnten bei verschiedenen Landschildkröten-Spezies nachgewiesen werden, am häufigsten bei Maurischen Landschildkröten. Sie wurden aber auch z.B. bei Griechischen und Russischen Landschildkröten, Panther- und Breit- randschildkröten ( $T$. marginata) und Indischen Sternschildkröten (Geochelone elegans) beschrieben.

\section{Intranukleäre Kokzidien}

Intranukleäre Kokzidien bei Landschildkröten (TINC) werden vor allem bei Schildkröten mit schweren systemischen Erkrankungen und unspezifischen klinischen Symptomen wie Lethargie und Anorexie, aber auch bei Tieren mit chronischer Rhinosinusitis nachgewiesen.

Am häufigsten werden intranukleäre Kokzidien bei tropischen Landschildkröten, z.B. Strahlenschildkröte, Pantherschildkröte, Sulawesi Landschildkröten, in den USA beschrieben. In den letzten Jahren wurden intranukleäre Kokzidien auch in Europa detektiert und scheinen auch bei europäischen Landschildkröten vorzukommen. Die Prävalenz scheint allerdings insgesamt sehr gering zu sein.

\section{Mischinfektionen}

Virusinfektionen sind häufig von Mykoplasmen begleitet, vor allem bei Infektionen mit Herpes- und Picornaviren. Wie die einzelnen Erreger untereinander agieren ist bisher unbekannt. Es wird vermutet, dass chronische Infektionen mit Mykoplasmen das Immunsystem des Wirtes schwächen und somit anfälliger für Viruserkrankungen machen. Weitere bakterielle Sekundärinfektionen mit fa-

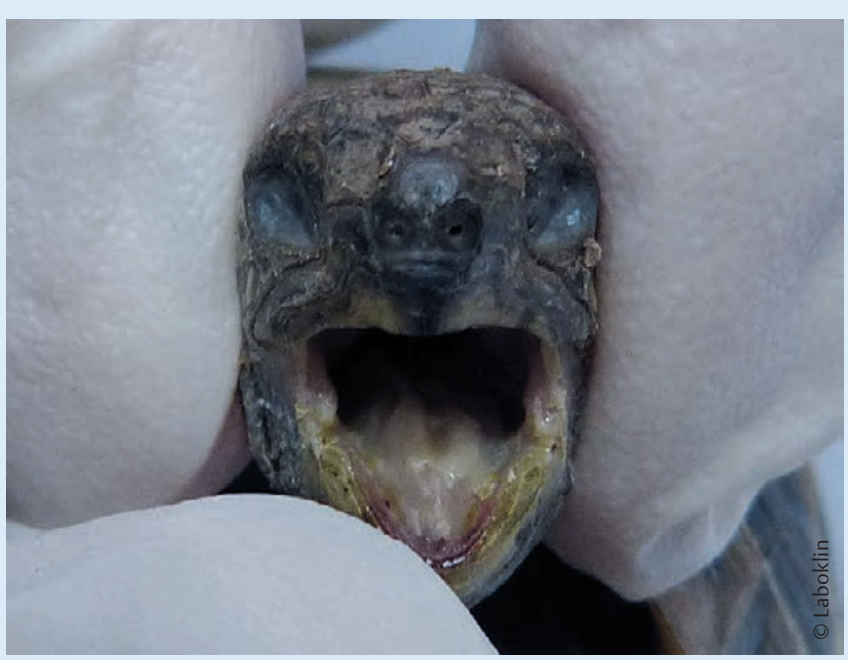

Abb. 3 Testudo hermanni mit eingefallenen Augen und Glossitis.

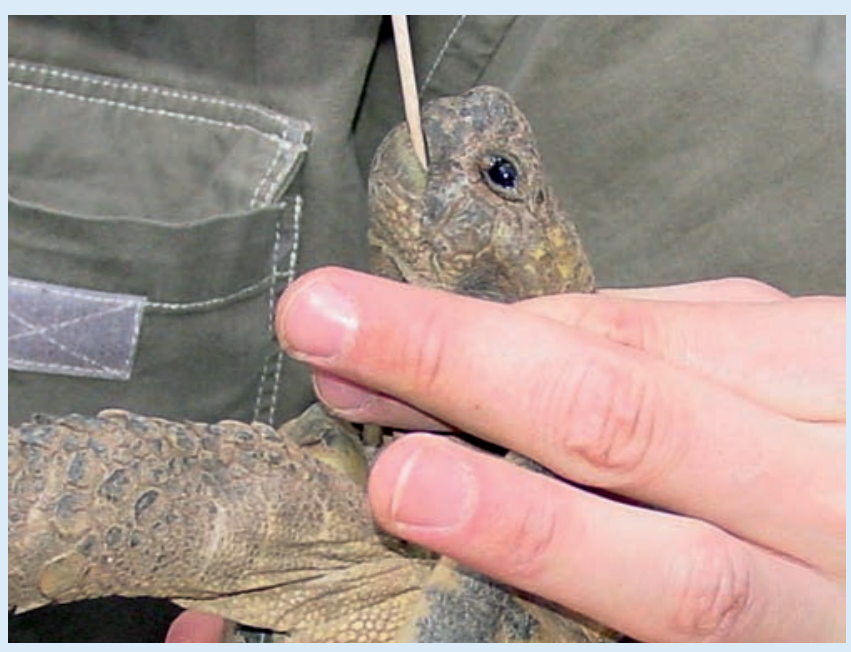

Abb. 4 Entnahme eines Rachentupfers bei einer Testudo hermanni. 
kultativ pathogenen Keimen sind möglich, wodurch die klinischen Symptome verschlimmert werden können. Durch die vielfältige bakterielle Flora im Rachen von Schildkröten, die sich vorwiegend aus gram-negativen Erregern zusammensetzt, ist eine reine bakteriologische Untersuchung daher oft wenig aussagekräftig.

\section{Diagnostik}

Für alle oben genannten Erreger stehen PCR zum Direktnachweis zur Verfügung. Geeignetes Probenmaterial sind Rachenabstriche ( $\triangleright$ Abb.4) und Nasenspülproben.

Die kulturelle Anzucht von Mykoplasmen wird routinemäßig kaum noch durchgeführt, da sie wegen ihres eingeschränkten Stoffwechsels nur sehr langsam (bis zu 6 Wochen) und nur auf speziellen Nährböden, z.B. SP4-Medium, wachsen.

\section{Therapie}

Die Behandlung von Mykoplasmeninfektionen sollte lokal und systemisch erfolgen. Geeignet sind Nasenspülungen mit physiologischer Kochsalzlösung oder verdünnter Antibiose und die systemische Gabe von z. B.

- Fluorchinolonen, z.B. Enrofloxacin: $10 \mathrm{mg} / \mathrm{kg}$ i.m. 1× tgl.; cave, kann lokale Reizung verursachen und ist schmerzhaft; als Nasenspülung: $50 \mathrm{mg} / 250 \mathrm{ml}$ steriles Wasser, 1-3 ml pro Nasenloch alle 1-2 Tage
- Makroliden (Clarithromycin): $15 \mathrm{mg} / \mathrm{kg}$ p.o. alle 2-3 Tage oder

- Tetrazyklinen, z.B. Doxycyclin: 5-10mg/ kg p.o. $1 \times$ tgl.

Dies bringt zwar keine Erregerfreiheit, aber eine Linderung der Symptome und eine Reduktion der Erregerausscheidung ist möglich.

Infektionen mit intranukleären Kokzidien können mit Toltrazuril behandelt werden. Dabei hängt der Therapieerfolg maßgeblich von einer frühzeitigen und ausreichend langen Behandlung ab.

- Toltrazuril (Baycox 5\%): $15 \mathrm{mg} / \mathrm{kg}$ p.o. alle 2 Tage für 30 Tage

Bei Herpesviren wird von Behandlungen mit Aciclovir oder verwandten Substanzen zur Besserung der Symptome berichtet:

- Aciclovir: $80 \mathrm{mg} / \mathrm{kg}$ p.o. $1 \times \operatorname{tgl}$.

Bei Viruserkrankungen wird der Einsatz von Paramunitätsinducern, z.B. Zyle$\mathrm{xis}^{\circledR}$, diskutiert, jedoch ist die Wirksamkeit bisher nicht bewiesen.

Allgemein sollten bei Erkrankungen die Umgebungstemperatur erhöht (innerhalb der bevorzugten Körpertemperatur) und die Haltungsbedingungen optimiert werden. Um die Ausbreitung im Bestand zu verhindern, sollten kranke Tiere isoliert werden (barrier nursing). Viele der genannten Erreger können persistierende Infektionen auch nach klinischer Besserung verursachen, sodass überlebende Tiere als Ansteckungsquelle für andere Tiere dienen könnten.

\section{Prophylaxe}

Labordiagnostische Untersuchungen im Rahmen von Quarantänemaßnahmen sind aufgrund der fehlenden Therapiemöglichkeiten und der Gefahr der schnellen Weiterverbreitung von Erregern im Bestand unerlässlich. Außerdem sollten die Tiere nach Spezies getrennt gehalten werden, da vermutet wird, dass verschiedene Spezies eine unterschiedliche Empfindlichkeit gegenüber den einzelnen Erregern aufweisen können. Subklinisch infizierte Tiere, die gesund erscheinen, können den Erreger somit schnell in einem Bestand verbreiten und bei empfindlichen Spezies zu schweren Erkrankungen bis hin zu plötzlichen Todesfällen führen.

Online zu finden unter

https://doi.org/10.1055/s-0043-119562

\section{Literatur}

Bei den Autoren erhältlich.

\section{Ekaterina Kolesnik}

PD Dr. Rachel E. Marschang,

Dipl. ECZM (herpetology)

Fachtierärztin für Mikrobiologie

Laboklin GmbH \& Co. KG

Steubenstr. 4

97688 Bad Kissingen

kolesnik@laboklin.com

marschang@laboklin.com 